\title{
A REFINEMENT OF STEIN FACTORIZATION AND DEFORMATIONS OF SURJECTIVE MORPHISMS*
}

\author{
STEFAN KEBEKUS ${ }^{\dagger}$ AND THOMAS PETERNELL ${ }^{\ddagger}$
}

\begin{abstract}
This paper is concerned with a refinement of the Stein factorization, and with applications to the study of deformations of morphisms. We show that every surjective morphism $f: X \rightarrow Y$ between normal projective varieties factors canonically via a finite cover of $Y$ that is éale in codimension one. This "maximally étale factorization" satisfies a strong functorial property.

It turns out that the maximally étale factorization is stable under deformations, and naturally decomposes an étale cover of the Hom-scheme into a torus and into deformations that are relative with respect to the rationally connected quotient of the target $Y$. In particular, we show that all deformations of $f$ respect the rationally connected quotient of $Y$.
\end{abstract}

Key words. Stein factorization, deformations of morphisms, maximal étale factorization, Homscheme.

AMS subject classifications. 14D15, 14J40, 14E99

1. Introduction and statement of results. Throughout this paper, we consider surjective morphisms between algebraic varieties and their deformations. To fix notation, we use the following assumption.

Assumption 1.1. $f: X \rightarrow Y$ will always denote a surjective holomorphic map between normal complex-projective varieties.

The main method that we introduce is a refinement of the Stein factorization: we show that $f$ factors canonically via a finite cover of $Y$ that is étale in codimension one. This "maximally étale factorization" satisfies a strong functorial property which is defined in Section 1.1 below and turns out to be stable under deformations of $f$.

We employ the maximally étale factorization for a study of the deformation space $\operatorname{Hom}(X, Y)$ and show that an étale cover of the Hom-scheme naturally decomposes into a torus and into deformations that are relative with respect to the maximally rationally connected fibration of the target $Y$. In particular, we show that all deformations of $f$ respect the rationally connected quotient of $Y$.

These result are summarized and properly formulated below.

1.1. The maximally étale factorization. Under the Assumptions 1.1, suppose that there exists a factorization $f$,

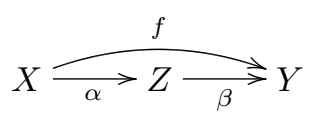

where $\beta$ is finite and étale in codimension 1, i.e. étale outside a set of codimension $\geq 2$.

\footnotetext{
*Received August 4, 2005; accepted for publication May 2, 2008.

${ }^{\dagger}$ Albert-Ludwigs-Universität Freiburg, Mathematisches Institut, Eckerstr. 1, 79104 Freiburg, Germany (stefan.kebekus@math.uni-freiburg.de).

${ }^{\ddagger}$ Institut für Mathematik, Universität Bayreuth, 95440 Bayreuth, Germany (thomas.peternell@ uni-bayreuth.de). Both authors were supported in part by the Forschungsschwerpunkt "Globale Methoden in der komplexen Analysis" of the Deutsche Forschungsgemeinschaft. A part of this paper was worked out while Stefan Kebekus visited the Korea Institute for Advanced Study. He would like to thank Jun-Muk Hwang for the invitation.
} 
DEFINITION 1.2. We say that a factorization $f=\beta \circ \alpha$ as in (1.1.1) is maximally étale if the following universal property holds: for any factorization $f=\beta^{\prime} \circ \alpha^{\prime}$, where $\beta^{\prime}: Z^{\prime} \rightarrow Y$ is finite and étale in codimension 1, there exists a morphism $\gamma: Z \rightarrow Z^{\prime}$ such that such that the following diagram commutes:

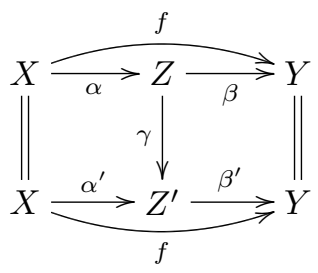

REMARK 1.3. It follows immediately from the definition that a maximally étale factorization of a given morphism $f$ is unique up to isomorphism if it exists. Theorem 4.1 describes the uniqueness in more detail.

The existence of the maximally étale factorization is established by the following theorem, which we prove in Section 3.

TheOREm 1.4. Let $f: X \rightarrow Y$ be a surjective morphism between normal projective varieties. Then there exists a maximally étale factorization.

We will later in Section 4 describe the maximally étale factorization in terms of the positivity of the push-forward sheaf $f_{*}\left(\mathcal{O}_{X}\right)$.

REMARK 1.5. We have already remarked that the maximally étale factorization yields a natural refinement of the Stein factorization. More precisely, we can say that a surjection $f: X \rightarrow Y$ of normal projective varieties decomposes as follows.

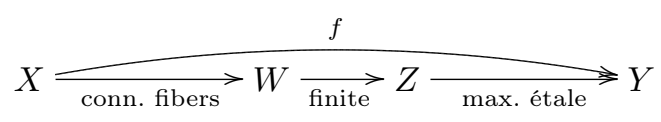

1.2. Stability of the factorization under deformations. Let $f=\alpha \circ \beta$, as in Diagram (1.1.1) denote the Stein factorization. If $f^{\prime}: X \rightarrow Y$ is any deformation of $f$, it is a classical fact that $f^{\prime}$ again factors via $\beta$ - see Section 2.1 for brief review. We will show that a similar, and somewhat stronger, property also holds for the maximally étale factorization. To formulate this stability result precisely, we introduce the following notation.

Notation 1.6. If $g: A \rightarrow B$ is any morphism between projective varieties, let $\operatorname{Hom}_{g}(A, B) \subset \operatorname{Hom}(A, B)$ be the connected component that contains $g$.

The stability result is then formulated as follows.

TheOrem 1.7. In the setup of Theorem 1.4, let $f=\beta \circ \alpha$ be the maximally étale factorization as in Diagram (1.1.1). Then the natural morphism between the reduced Hom-spaces,

$$
\begin{array}{rlc}
\eta: \operatorname{Hom}_{\alpha}(X, Z)_{\mathrm{red}} & \rightarrow & \operatorname{Hom}_{f}(X, Y)_{\mathrm{red}} \\
\alpha^{\prime} & \mapsto & \beta \circ \alpha^{\prime}
\end{array}
$$


is proper and surjective. The induced morphism $\tilde{\eta}$ between the normalizations is étale. If $f^{\prime} \in \operatorname{Hom}_{f}(X, Y)$ is any deformation of $f$, then $f^{\prime}$ factors via $Z$, and has $Z$ as maximally étale factorization.

We prove Theorem 1.7 in Section 5.

1.3. Decomposition of the Hom-scheme. We recall the main result of [HKP06], where deformations of morphisms with non-uniruled targets were studied. Using the language of Section 1.1, this is formulated as follows.

ThEOREM 1.8 ([HKP06, thm. 1.2]). Under the Assumptions 1.1, suppose that $Y$ is not covered by rational curves. If

$$
X \underset{\alpha}{\longrightarrow} \underset{\beta}{\longrightarrow} Y
$$

denotes the maximally étale factorization, and if $\operatorname{Aut}^{0}(Z)$ is the maximal connected subgroup of the automorphism group of $Z$, then $\operatorname{Aut}^{0}(Z)$ is an Abelian variety, and the natural morphism

$$
\operatorname{Aut}^{0}(Z) / \operatorname{Aut}(Z / Y) \cap \operatorname{Aut}^{0}(Z) \rightarrow \operatorname{Hom}_{f}(X, Y)
$$

is an isomorphism of schemes.

In particular, all deformations of surjective morphisms $X \rightarrow Y$ are unobstructed, and the associated components of $\operatorname{Hom}_{f}(X, Y)$ are smooth Abelian varieties.

At the other extreme, if $Y$ is rationally connected, partial descriptions of the Homscheme are known - the results of [HM03, thm. 1] and [HM04, thm. 3] assert that whenever $Y$ is a Fano manifolds of Picard-number 1 whose variety of minimal rational tangents is finite, or not linear, then all deformations of $f$ come from automorphisms of $Y$. This covers all examples of Fano manifolds of Picard-number one that one encounters in practice.

If $Y$ is covered by rational curves, but not rationally connected, we consider the maximally rationally connected fibration $q_{Y}: Y \rightarrow Q_{Y}$ which is explained in more detail in Section 2.2. Using the maximally étale factorization, we will show that an étale cover of $\widetilde{\operatorname{Hom}}_{f}(X, Y)$, the normalization of the space $\operatorname{Hom}_{f}(X, Y)$, can be decomposed into a torus and a space of deformations that are relative with respect $q_{Y}$. We recall the notion of a relative deformation first.

Notation 1.9. We call the subvariety

$$
H_{\mathrm{vert}}^{f}:=\left\{f^{\prime} \in \operatorname{Hom}_{f}(X, Y)_{\text {red }} \mid q_{Y} \circ f^{\prime}=q_{Y} \circ f\right\}
$$

the "space of relative deformations of $f$ over $q_{Y}$ ".

The following theorem will then be shown in Section 7.

THEOREM 1.10. Under the Assumption 1.1, let

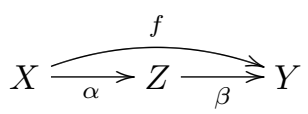

be the maximally étale factorization, and $T \subset \operatorname{Aut}^{0}(Z)$ a maximal compact Abelian subgroup. Then the following holds: 
(1.10.1) There exists a normal variety $\tilde{H}$ and an étale morphism

$$
T \times \tilde{H} \rightarrow \widetilde{\operatorname{Hom}}_{f}(X, Y)
$$

that maps $\{e\} \times \tilde{H}$ to the preimage of $H_{\mathrm{vert}}^{f}$.

(1.10.2) If $Y$ is smooth or if $f$ is itself maximally étale, then $\{e\} \times \tilde{H}$ surjects onto the preimage of $H_{\mathrm{vert}}^{f}$.

In the setup of Theorem 1.10, it need not be true that $\operatorname{Aut}^{0}(Z)$ is itself an Abelian variety. The existence of a maximal compact Abelian subgroup $T \subset \operatorname{Aut}^{0}(Z)$ is briefly discussed in Fact 6.1 on page 379 below.

REMARK 1.11. The assertion of Theorem 1.10 is weaker than Theorem 1.8 in the sense that it does not make any statement about the scheme-structure of $\operatorname{Hom}_{f}(X, Y)$. The reason is that the maximal rationally connected fibration $q_{Y}$ need not be a morphism, and that there is no good deformation space for rational maps between fixed varieties. Theorem 1.10 can certainly be straightened if one assumes additionally that $q_{Y}$ is regular.

Acknowledgments. The authors would like to thank Ivo Radloff and Eckhard Viehweg for a number of discussions. It was in these discussion that the notion of "maximally étale" evolved.

2. Known Facts. The proofs of our main results rely on a number of facts scattered throughout the literature. For the reader's convenience, we have gathered these here.

2.1. Stability of Stein factorization under deformation. Consider the Stein factorization of $f$,

$$
X \underset{g: \text { conn. fibers }}{\stackrel{f}{\longrightarrow}} W_{0} \underset{h: \text { finite }}{\longrightarrow} Y .
$$

We will later need to know that any deformation of $f$ still has $g: X \rightarrow W_{0}$ as Stein factorization. While this is probably well-understood, we were unable to find a good reference for the universal properties of Stein factorization, and include a full proof.

Proposition 2.1 (Stability of Stein factorization under deformation). The canonical composition morphism

$$
\begin{array}{clc}
\nu: \operatorname{Hom}_{h}\left(W_{0}, Y\right) & \rightarrow & \operatorname{Hom}_{f}(X, Y) \\
h^{\prime} & \mapsto & h^{\prime} \circ g
\end{array}
$$

is bijective. In particular, the morphism between the normalized Hom-schemes is isomorphic.

The proof of Proposition 2.1, which we give below, makes use of the following two lemmas, which assert that a deformation of a morphism with connected fibers does not change the fibers, and that a surjective morphism between normal spaces is determined up to isomorphism by its set-theoretical fibers.

Lemma 2.2 (Invariance of fibers under deformation). Let $T$ be a smooth curve and $\left(f_{t}\right)_{t \in T}: X \rightarrow Y$ be a family of surjective morphisms between projective varieties. Assume that for all $t \in T$, the map $f_{t}$ has connected fibers. Then the set-theoretical 
fibers of $f_{t}$ are independent of $t$. More precisely, for all $x \in X$ and all $s, t \in T$, we have

$$
f_{t}^{-1} f_{t}(x)=f_{s}^{-1} f_{s}(x)
$$

Proof. Choose an ample line bundle $L \in \operatorname{Pic}(X)$. Observe that two points $x, y \in$ $X$ are contained in the same $f_{t}$-fiber if and only if there exists a curve $C \subset X$ that contains both $x$ and $y$, and satisfies $c_{1}\left(f_{t}^{*}(L)\right) . C=0$. Likewise, $x$ and $y$ are in the same $f_{s}$-fiber if and only if there is a curve $C \subset X$ with $x, y \in C$ and $c_{1}\left(f_{s}^{*}(L)\right) . C=0$.

On the other hand, since $f_{t}$ and $f_{s}$ are homotopic, the Chern classes of the pullback bundles agree,

$$
c_{1}\left(f_{t}^{*}(L)\right)=c_{1}\left(f_{s}^{*}(L)\right) .
$$

This shows the claim. $\square$

Lemma 2.3. Let $f_{1}: X \rightarrow W_{1}$ and $f_{2}: X \rightarrow W_{2}$ be two surjective morphisms between normal spaces. If for all $x \in X$ the set-theoretical fibers of $f_{1}$ and $f_{2}$ agree, i.e. if $f_{1}^{-1}\left(f_{1}(x)\right)=f_{2}^{-1}\left(f_{2}(x)\right)$, then there exists a commutative diagram as follows.

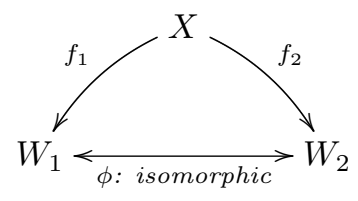

Proof. The morphisms $f_{1}, f_{2}$ give rise to a morphism from $\iota: X \rightarrow W_{1} \times W_{2}$, $\iota(x)=\left(f_{1}(x), f_{2}(x)\right)$, and we obtain a commutative diagram as follows.

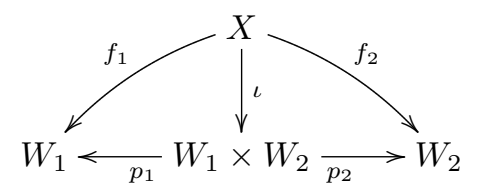

The assumptions that the fibers of $f_{1}$ and $f_{2}$ agree implies that the restrictions $\left.p_{i}\right|_{\iota(X)}$ of the morphisms $p_{1}$ and $p_{2}$ to the image $\iota(X)$ are bijective. Since we are working over $\mathbb{C}$, Zariski's main theorem then implies that the restrictions $\left.p_{1}\right|_{\iota(X)}$ and $\left.p_{2}\right|_{\iota(X)}$ are even isomorphic. We can therefore view $\iota(X)$ as the graph of an invertible morphism $\phi:=\left.p_{2}\right|_{\iota(X)} \circ\left(\left.p_{1}\right|_{\iota(X)}\right)^{-1}$, which yields the claim.

Proof of Proposition 2.1. The injectivity of $\nu$ is obvious because $g$ is surjective. Since $\operatorname{Hom}_{f}(X, Y)$ is connected, to prove surjectivity, it suffices to show that any morphism $\gamma_{f}: T \rightarrow \operatorname{Hom}_{f}(X, Y)$ from a smooth irreducible curve $T$ can be lifted to a curve $\gamma_{h}: T \rightarrow \operatorname{Hom}_{h}\left(W_{0}, Y\right)$ such that $\gamma_{f}=\nu \circ \gamma_{h}$.

To this end, let

$$
\begin{array}{ccc}
F: \quad X \times T & \rightarrow & Y \times T \\
(x, t) & \mapsto & \left(f_{t}(x), t\right)
\end{array}
$$

be the proper product morphism of the universal map and the identity, and consider the Stein factorization

$$
X \times T \underset{\text { G: conn. fibers }}{\longrightarrow} W \underset{H: \text { finite }}{\longrightarrow} Y \times T .
$$


Lemma 2.2 on the invariance of fibers implies that the morphisms $g \times I d_{T}: X \times T \rightarrow$ $W_{0} \times T$ and $G$ have the same fibers. Lemma 2.3 then asserts that there exists an isomorphism $\phi$ such that the factorization (2.3.1) extends to a commutative diagram as follows.

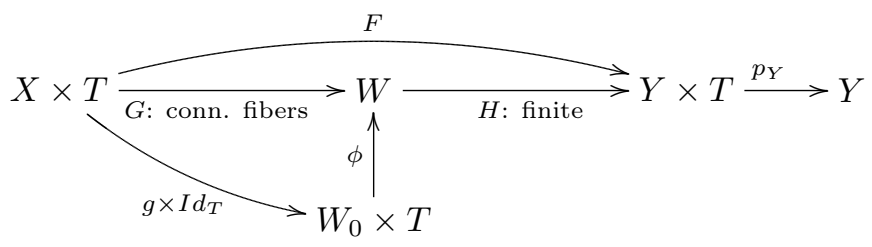

We use Diagram (2.3.2) to define the morphism $\gamma_{h}: T \rightarrow \operatorname{Hom}_{h}\left(W_{0}, Y\right)$ by

$$
\begin{array}{rlc}
\gamma_{h}(t): W_{0} & \rightarrow & Y \\
w & \mapsto & p_{Y}(H(\phi(w, t)))
\end{array}
$$

It follows then from the commutativity of Diagram (2.3.2) that $\gamma_{f}=\nu \circ \gamma_{h}$. This proves Proposition 2.1.

2.2. The rational quotient. Recall from [Kol96, chap. IV] or [Deb01, sec. 4] that an irreducible projective variety $X$ is rationally connected if any two sufficiently general points can be joined by a single rational curve. Moreover $X$ is rationally chain connected if two general points can be joined by a connected chain of rational curves.

REMARK 2.4. If $X$ is smooth, then $X$ is rationally connected if and only if $X$ is rationally chain connected [Kol96]. If $X$ is singular, this need no longer be true. For instance, if $X$ is the cone over an elliptic curve, then $X$ is of course rationally chain connected, but not rationally connected.

One of the most important features of uniruled varieties is the existence of a rationally connected quotient, introduced by Campana and Kollár-Miyaoka-Mori.

DeFinition 2.5. Let $V$ be a normal variety and $r_{V}: V \rightarrow R_{V}$ a dominant rational map to a normal variety. The map $r_{V}$ is called a maximal rationally chain connected fibration, if for all very general points $v \in V$, the closure of the fiber through $v$,

$$
R(v):=\overline{r_{V}^{-1}\left(r_{V}(v)\right)}
$$

is the largest rationally chain connected subvariety of $V$ that contains $v$.

The existence of a maximal rationally chain connected fibration is established by Campana (even in the Kähler case) and Kollár-Miyaoka-Mori, see [Kol96] and [Deb01]. Campana uses the notation "rational quotient".

FACT 2.6. Let $V$ be a normal projective variety. Then there exists a maximal rationally chain connected fibration $r_{V}: V \rightarrow R_{V}$, with the additional property that the quotient map $r_{V}$ is almost holomorphic, i.e. there exists a dense open subset $V^{0} \subset V$ such that the restriction $\left.r_{V}\right|_{V^{0}}$ is a proper morphism.

Notice that Kollár-Miyaoka-Mori [Kol96] and Debarre [Deb01] already put the property to be almost holomorphic into the definition of a maximal rationally chain connected fibration. We include this into the next notion. 
We will need to consider a different variant of a rational quotient coming from the fact that for singular varieties rational connectedness and rational chain connectedness do not coincide.

Definition 2.7. Let $V$ be a normal variety and $q_{V}: V \rightarrow Q_{V}$ a dominant rational map to a normal variety. The map $q_{V}$ is called a maximal rationally connected fibration, if for all very general points $v \in V$, the closure of the fiber through $v$,

$$
R(v):=\overline{q_{V}^{-1}\left(q_{V}(v)\right)},
$$

is the largest rationally connected subvariety of $V$ that contains $v$.

Proposition 2.8. If $V$ is a normal projective variety, then there exists a maximal rationally connected fibration $q_{V}: V \rightarrow Q_{V}$.

Proof. Let $\eta: \tilde{V} \rightarrow V$ be a desingularization, and $r_{\tilde{V}}: \tilde{V} \rightarrow R_{\tilde{V}}$ a maximal rationally chain connected fibration. Then set $Q_{V}=R_{\tilde{V}}$ and $q_{V}:=r_{\tilde{V}} \circ \eta^{-1}$.

Notice that there is a factorization $Q_{V} \rightarrow R_{V}$. Of course both fibration are unique up to birational equivalence, so that we speak of "the" maximal rationally (chain) connected fibration.

REMARK 2.9. If $V$ is singular, a maximal rationally connected fibration of $V$ is not necessarily the maximal rationally chain fibration. E.g., if $X$ is the cone over an elliptic curve, then the maximal rationally connected fibration maps to the elliptic curve, whereas the maximal rationally chain connected fibration maps to a point. Further,the maximal rationally connected fibration cannot not necessarily be taken to be almost holomorphic.

It is a crucial fact shown by Graber, Harris and Starr that the base of a maximal rationally chain connected fibration, hence also of a maximal rationally connected fibration is itself not uniruled.

FACT 2.10 ([GHS03, cor. 1.4]). If $q_{V}: V \rightarrow Q_{V}$ is a maximal rationally (chain) connected fibration, then $Q_{V}$ is not uniruled.

The maximal rationally (chain) connected fibration described in the literature is determined only up to birational equivalence. It is, however, easy to see that there is a canonical choice.

Proposition 2.11. Let $V$ be a normal projective variety. Then there exists a canonical maximal rationally (chain) connected fibration $q_{V}: V \rightarrow Q_{V}$, with the following property: the automorphism group $\operatorname{Aut}(V)$ stabilizes the indeterminacy locus of $q_{V}$, and has a natural action on $Q_{V}$ such that $q_{V}$ is equivariant wherever it is defined.

Proof. Let $q: V \rightarrow Q$ be any maximal rationally (chain) fibration. The universal property of the cycle space than yields a rational map as follows:

$$
\begin{array}{cccc}
q_{V}^{\prime}: & V & \rightarrow & \text { Chow }(V) \\
& v & \mapsto & R(v) .
\end{array}
$$

This construction has two important features. For one, observe that the morphism $q_{V}^{\prime}$ is independent of the particular choice of the rationally connected quotient $q$. Secondly, if $x \in V$ is a very general point, and $g \in \operatorname{Aut}(V)$ is any automorphism, then $g(R(x))$ is again rationally (chain) connected. In particular, we have that $g(R(x)))=$ 
$R(g(x))$. This already shows that the natural action of $\operatorname{Aut}(V)$ on $\operatorname{Chow}(V)$ stabilizes the image of $q_{V}^{\prime}$ and makes $q_{V}^{\prime}$ equivariant. The proof is thus finished if we let $Q_{V}$ be the normalization of the closure of the image of $q_{V}^{\prime}$, and $q_{V}: V \rightarrow Q_{V}$ be the lifting that comes from the universal property of the normalization.

Notation 2.12. For the rest of this paper, if we discuss "the" maximal rationally (chain) connected fibration of a variety, we always mean the canonic construction given in Proposition 2.11.

We will later need to consider subsheaves of the tangent sheaf $T_{V}$ that are relative over the rationally connected quotient wherever this is well-defined.

DeFINITION 2.13. Let $V$ be a normal projective variety, and let $q_{V}: V \rightarrow Q_{V}$ be the rationally connected quotient. Further, suppose that $C$ is a normal variety and $\iota: C \rightarrow V$ a morphism whose image is not contained in the singular locus of $V$, and not contained in the indeterminacy locus of $q_{V}$. If $\mathcal{F} \subset \iota^{*}\left(T_{V}\right)$ is a reflexive subsheaf of the pull-back of the tangent sheaf, we say that $\mathcal{F}$ is vertical with respect to the rationally connected quotient, if $\mathcal{F}$ is contained in $\iota^{*}\left(T_{V \mid Q_{V}}\right)$ at the general point of $C$.

Likewise, a morphism of reflexive sheaves $\mathcal{F} \rightarrow \iota^{*}\left(T_{V}\right)$ is vertical with respect to the rationally connected quotient if the double dual of its image is. An infinitesimal deformation of $\iota$, i.e. an element $\sigma \in \operatorname{Hom}\left(\iota^{*}\left(\Omega_{V}^{1}\right), \mathcal{O}_{C}\right)$, is vertical if the restriction of $\sigma$ to general points of $C$ corresponds to a section in $\iota^{*}\left(T_{V \mid Q_{V}}\right)$.

2.3. General curves in projective varieties. We will later need to consider the Harder-Narasimhan filtration of the tangent sheaf $T_{X}$. By Mehta-Ramanathan's theorem, it suffices to discuss the filtration of the restriction to a general complete intersection curve, whose definition we recall now.

Definition 2.14. If $X$ is normal, we consider general complete intersection curves in the sense of Mehta-Ramanathan, $C \subset X$. These are reduced, irreducible curves of the form $C=H_{1} \cap \cdots \cap H_{\operatorname{dim} X-1}$, where the $H_{i} \in\left|m_{i} \cdot H\right|$ are general, the $L_{i} \in \operatorname{Pic}(X)$ are ample and the $m_{i} \in \mathbb{N}$ large enough, so that the Harder-NarasimhanFiltration of $T_{X}$ commutes with restriction to $C$. If the $L_{i}$ are chosen, we also call $C$ $a$ general complete intersection curve with respect to $\left(L_{1}, \ldots, L_{\operatorname{dim} X-1}\right)$.

We refer to [Fle84] and [Lan04] for a discussion and an explicit bound for the $m_{i}$ that appear in Definition 2.14.

If $X$ is a normal variety, $q_{X}: X \rightarrow Q_{X}$ the maximal rationally connected fibration and $C \subset X$ a general complete intersection curve, then $C$ intersects neither the singular locus of $X$, nor the indeterminacy locus of $q_{X}$. It makes therefore sense ask if a subsheaf $\left.\mathcal{F}_{C} \subset T_{X}\right|_{C}$ is vertical with respect to the rationally connected quotient. The following important criterion is a refinement of Miyaoka's characterization of uniruled varieties. It appeared first implicitly in [Kol92, 9.0.3], but see [KST07, rem. 4.8].

FACT 2.15 ([KST07, cor. 1.4]). Let $X$ be a normal projective variety with maximal rationally connected fibration $q_{X}: X \rightarrow Q_{X}$. If $C \subset X$ is a general complete intersection curve and $\mathcal{F}_{C} \subset T_{X} \mid C$ a locally free and ample subsheaf, then $\mathcal{F}_{C} \subset T_{X / Q_{X}} \mid C \cdot \square$ 
2.4. Finite morphisms. Let $f: X \rightarrow Y$ be a surjective, finite morphism between normal varieties. The push-forward of the structure sheaf $f_{*}\left(\mathcal{O}_{X}\right)$ is then a torsion free sheaf on $X$, which is locally free where $f$ is flat, i.e. away from the singularities of $X$ and $Y$. Much of our argumentation is based on an analysis of the positivity properties of $f_{*}\left(\mathcal{O}_{X}\right)$.

Notation 2.16. Let $X_{\text {Sing }}$ and $Y_{\text {Sing }}$ denote the singular loci, and set

$$
\begin{aligned}
& X^{0}=X \backslash\left(X_{\text {Sing }} \cup f^{-1}\left(Y_{\text {Sing }}\right)\right) \\
& Y^{0}=Y \backslash\left(Y_{\text {Sing }} \cup f\left(X_{\text {Sing }}\right)\right)=f\left(X^{0}\right) .
\end{aligned}
$$

Then $\operatorname{codim} X \backslash X^{0}=\operatorname{codim} Y \backslash Y^{0} \geq 2$.

FACT 2.17. The trace morphism $t r: f_{*}\left(\mathcal{O}_{X^{0}}\right) \rightarrow \mathcal{O}_{Y^{0}}$ gives rise to a splitting

$$
f_{*}\left(\mathcal{O}_{X^{0}}\right) \cong \mathcal{O}_{Y^{0}} \oplus \mathcal{E}_{f}^{\vee}
$$

where $\mathcal{E}_{f}^{\vee}$ is a locally free sheaf on $Y^{0}$. Let $\mathcal{E}_{f}$ be the dual ${ }^{1}$ of $\mathcal{E}_{f}^{\vee}$.

The following result on the positivity of $\mathcal{E}_{f}$ appeared only recently. We have however learned from E. Viehweg that it is implicitly contained in much older works of Fujita.

FACT 2.18 ([PS00, Thm. A of the appendix by Lazarsfeld]). Let $C \subset Y^{0}$ be a complete curve that is not contained in the branch locus of $f$. Then $\left.\mathcal{E}_{f}\right|_{C}$ is a nef vector bundle on $C$. It has degree 0 if and only if $f$ is unbranched along $C$.

Corollary 2.19. If $C \subset Y$ is a general complete intersection curve, then $\left.f_{*}\left(\mathcal{O}_{X}\right)\right|_{C}$ is of degree 0 if and only if $f$ is étale in codimension 1.

As a consequence of the projection formula, $f_{*} f^{*}(\mathcal{F})=f_{*}\left(\mathcal{O}_{X}\right) \otimes \mathcal{F}$, we obtain that if $\mathcal{F}$ is any coherent sheaf on $Y$, then there is a natural direct sum decomposition

$$
H^{0}\left(X^{0}, f^{*}(\mathcal{F})\right) \cong H^{0}\left(Y^{0}, \mathcal{F}\right) \oplus \operatorname{Hom}_{Y^{0}}\left(\mathcal{E}_{f},\left.\mathcal{F}\right|_{Y^{0}}\right)
$$

Notation 2.20. In the setup of this section, if $\sigma \in H^{0}\left(X, f^{*}(\mathcal{F})\right)$, let $\sigma=\sigma_{f}^{\prime}+\sigma_{f}^{\prime \prime}$ be the decomposition that is associated with the splitting (2.19.1).

3. Existence of a max. étale factorization, Proof of Theorem 1.4. We will in this section prove the existence of a maximally étale factorization for surjective morphisms between normal projective varieties. Since the proof is somewhat long, we subdivide it into a number of steps. We maintain the notation and the assumptions of Theorem 1.4 throughout.

The strategy of proof follows [HKP06]: we construct the factorization using a suitable subsheaf of $f_{*}\left(\mathcal{O}_{X}\right)$.

3.1. Reduction to the case of a finite morphism. Using the Stein factorization of the morphism $f$, we can assume without loss of generality that $f$ is actually finite.

\footnotetext{
${ }^{1}$ The use of the 'dual' follows historical conventions. We use it to be consistent with the literature we cite.
} 
3.2. The Harder-Narasimhan-Filtration. Choose an ample line bundle $H \in$ $\operatorname{Pic}(Y)$, and consider the associated Harder-Narasimhan-Filtration of $f_{*}\left(\mathcal{O}_{X}\right)$,

$$
0=\mathcal{F}_{0} \subset \mathcal{F}_{1} \subset \cdots \subset \mathcal{F}_{r-1} \subset \mathcal{F}_{r}=f_{*}\left(\mathcal{O}_{X}\right) .
$$

Lemma 3.1. The degree of $\mathcal{F}_{1}$ with respect to $H$ is zero,

$$
\operatorname{deg}_{H}\left(\mathcal{F}_{1}\right):=c_{1}\left(\mathcal{F}_{1}\right) \cdot c_{1}(H)^{\operatorname{dim} Y-1}=0 .
$$

If $\mathcal{G} \subset f_{*}\left(\mathcal{O}_{X}\right)$ is any coherent subsheaf with $\operatorname{deg}_{H}(\mathcal{G})=0$, then $\mathcal{G} \subset \mathcal{F}_{1}$.

Proof. Consider the splitting $f_{*}\left(\mathcal{O}_{X}\right)=\mathcal{O}_{Y} \oplus \mathcal{E}^{*}$. Since a general complete intersection curve $C \subset Y$ is not contained in the branch locus of $f$, Lazarsfeld's result, Fact 2.18, asserts that $\left.\mathcal{E}^{*}\right|_{C}$ is an anti-nef vector bundle. This in turn implies that no subsheaf of $f_{*}\left(\mathcal{O}_{X}\right)$ has positive degree. Since $\mathcal{O}_{Y} \subset f_{*}\left(\mathcal{O}_{X}\right)$ is a subsheaf of $\operatorname{deg}_{H}\left(\mathcal{O}_{Y}\right)=0$, either $f_{*}\left(\mathcal{O}_{X}\right)$ is $H$-semistable and $\operatorname{deg}_{H} f_{*}\left(\mathcal{O}_{X}\right)=0$, or the degree of the maximally destabilizing subsheaf $\mathcal{F}_{1}$ is zero. The first statement thus follows.

The second statement is void if $f_{*}\left(\mathcal{O}_{X}\right)$ is semistable (so that $\mathcal{F}_{1}=f_{*}\left(\mathcal{O}_{X}\right)$ ). We can thus assume that $f_{*}\left(\mathcal{O}_{X}\right)$ is not semistable, and that we are given a coherent subsheaf $\mathcal{G} \subset f_{*}\left(\mathcal{O}_{X}\right)$ with $\operatorname{deg}_{H}(\mathcal{G})=0$. Consider the image of $\mathcal{F}_{1}$ and $\mathcal{G}$ under the addition map,

$$
+: \mathcal{F}_{1} \oplus \mathcal{G} \rightarrow f_{*}\left(\mathcal{O}_{X}\right) .
$$

The image sheaf again has non-negative degree and must therefore be contained in the maximally destabilizing subsheaf $\mathcal{F}_{1}$. This proves that $\mathcal{G} \subset \mathcal{F}_{1}$.

Lemma 3.2. The $\mathcal{O}_{Y}$-algebra structure on $f_{*}\left(\mathcal{O}_{X}\right)$ induces on $\mathcal{F}_{1}$ the structure of a sheaf of $\mathcal{O}_{Y}$-subalgebras.

Proof. Since $\mathcal{F}_{1}$ is a sheaf of $\mathcal{O}_{Y}$-modules which contains $\mathcal{O}_{Y}$, it solely remains to verify that $\mathcal{F}_{1}$ is closed under the multiplication map

$$
m: f_{*}\left(\mathcal{O}_{X}\right) \otimes f_{*}\left(\mathcal{O}_{X}\right) \rightarrow f_{*}\left(\mathcal{O}_{X}\right) .
$$

In other words, we need to check that the associated map

$$
m^{\prime}: \mathcal{F}_{1} \otimes \mathcal{F}_{1} \rightarrow f_{*}\left(\mathcal{O}_{X}\right) / \mathcal{F}_{1}
$$

is constantly zero. Again, if $\mathcal{F}_{1}=f_{*}\left(\mathcal{O}_{Y}\right)$, there is nothing to show. Otherwise, observe that $\mathcal{F}_{1} \otimes \mathcal{F}_{1}$ is semistable with slope $\mu\left(\mathcal{F}_{1} \otimes \mathcal{F}_{1}\right)=0$ so that $f_{*}\left(\mathcal{O}_{X}\right) / \mathcal{F}_{1}$ contains a subsheaf $\mathcal{G}$ with $\operatorname{deg}_{H} \mathcal{G}=0$. By Lemma 3.1 this subsheaf must vanish, hence $m^{\prime}=0$.

3.3. Construction of the factorization, end of proof. Since $\mathcal{F}_{1}$ is a coherent sheaf of $\mathcal{O}_{Y}$-algebras, the morphism $f$ now automatically factorizes via $Z:=\operatorname{Specan}\left(\mathcal{F}_{1}\right)$.

$$
X \underset{\alpha}{\stackrel{f}{\longrightarrow} \underset{\beta}{\longrightarrow}} Y
$$

Since $\beta$ is proper and affine, it is clear that it must be finite. We will now show that $Z$ is normal, that $\beta$ is étale in codimension 1 , and that it is indeed maximally étale. 
Lemma 3.3. The intermediate variety $Z$ is normal.

Proof. Let $\eta: \tilde{Z} \rightarrow Z$ be the normalization morphism. The universal property of normalization then yields a further factorization

$$
X \underset{\tilde{\alpha}}{\stackrel{f}{\longrightarrow} \underset{\eta}{\longrightarrow} \underset{\beta}{\longrightarrow}} Y .
$$

Accordingly, we obtain a sequences of subsheaves of $\mathcal{O}_{Y}$-algebras,

$$
\mathcal{F}_{1}=\beta_{*}\left(\mathcal{O}_{Z}\right) \subset(\beta \circ \eta)_{*}\left(\mathcal{O}_{\tilde{Z}}\right) \subset f_{*}\left(\mathcal{O}_{X}\right) .
$$

Since $\eta$ is isomorphic away from a proper subset, the quotient $\mathcal{Q}:=(\beta \circ \eta)_{*}\left(\mathcal{O}_{\tilde{Z}}\right) / \mathcal{F}_{1}$ either vanishes, or is a torsion sheaf. But since $\mathcal{F}_{1}$ is saturated in $f_{*}\left(\mathcal{O}_{X}\right)$, the quotient $\mathcal{Q}$ cannot be non-zero torsion. This shows that $(\beta \circ \eta)_{*}\left(\mathcal{O}_{\tilde{Z}}\right)=\mathcal{F}_{1}$ and therefore $Z=\tilde{Z}$. $\mathrm{\square}$

LEMMA 3.4. The morphism $\beta$ is étale in codimension 1.

Proof. By Corollary 2.19, to prove the assertion, it is equivalent to show $\operatorname{deg}\left(\left.\beta_{*}\left(\mathcal{O}_{Z}\right)\right|_{C}\right)=0$. Since $\beta_{*}\left(\mathcal{O}_{Z}\right)=\mathcal{F}_{1}$, and since $\operatorname{deg}\left(\left.\beta_{*}\left(\mathcal{O}_{Z}\right)\right|_{C}\right)=\operatorname{deg}_{H}\left(\mathcal{F}_{1}\right)$, this follows from the first statement of Lemma 3.1.

Lemma 3.5. The factorization (3.2.1) is maximally étale.

Proof. Let $f=\beta^{\prime} \circ \alpha^{\prime}$ be any factorization via an intermediate variety $Z^{\prime}$ which is étale in codimension 1 over $Y$. The push-forward $\mathcal{G}:=\beta_{*}^{\prime}\left(\mathcal{O}_{Z^{\prime}}\right) \subset f_{*}\left(\mathcal{O}_{X}\right)$ is then a

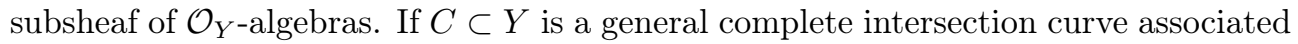
with the polarization $H$, then $\mathcal{G}$ is locally free along $C$, and Fact 2.18 asserts that $\operatorname{deg}\left(\left.\mathcal{G}\right|_{C}\right)=0$. In other words, we have that $\operatorname{deg}_{H}(\mathcal{G})=0$, and the second statement of Lemma 3.1 implies that $\mathcal{G} \subset \mathcal{F}_{1}=\beta_{*}\left(\mathcal{O}_{Z}\right)$.

This ends the proof of Theorem 1.4.

REMARK 3.6. If $X$ and $Y$ are smooth, the maximally étale factorization

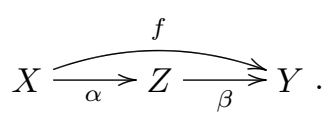

can more easily be constructed as follows: The subgroup $f_{*}\left(\pi_{1}(X)\right) \subset \pi_{1}(Y)$ has finite index, and therefore determines a finite étale cover $g: \tilde{Y} \rightarrow Y$ such that $f$ factors via $g$. As the map $\pi_{1}(X) \rightarrow \pi_{1}(\tilde{Y})$ must necessarily be onto, the factorization via $g$ is maximal.

4. Characterization of the maximally étale factorization. We will later need to characterize the maximally étale factorization among all factorizations in terms of positivity properties of the push-forward sheaf $\beta_{*}\left(\mathcal{O}_{Z}\right)$. The construction of the maximally étale factorization in the previous section almost immediately yields the following.

TheOrem 4.1. Let $f: X \rightarrow Y$ be a surjection between normal projective varieties with maximally étale factorization

$$
X \underset{\alpha}{\stackrel{f}{\longrightarrow}} \underset{\beta}{\longrightarrow} Y .
$$


Let $H \in \operatorname{Pic}(Y)$ be an arbitrary polarization and $C \subset Y$ an associated general complete intersection curve. Then

(4.1.1) the push-forward $\beta_{*}\left(\mathcal{O}_{Z}\right)$ is the maximally destabilizing subsheaf of $f_{*}\left(\mathcal{O}_{X}\right)$ with respect to the polarization $H$, and $Z=\operatorname{Specan}\left(\beta_{*}\left(\mathcal{O}_{Z}\right)\right)$, and

(4.1.2) if we set $\mathcal{Q}:=f_{*}\left(\mathcal{O}_{X}\right) / \beta_{*}\left(\mathcal{O}_{Z}\right)$, then $\left.\mathcal{Q}^{\vee}\right|_{C}$ is an ample vector bundle on $C$.

Proof. Statement (4.1.1) is a direct corollary to the proof of Theorem 1.4. In fact, in Section 3, we have chosen one particular polarization $H \in \operatorname{Pic}(Y)$, and constructed $Z$ as the Specan of the maximally destabilizing subsheaf $\mathcal{F}_{1} \subset f_{*}\left(\mathcal{O}_{X}\right)$. While $\mathcal{F}_{1}$ could a priori depend on the choice of $H$, the universal property of the maximally étale factorization shows that it actually does not: if $\beta^{\prime}: Z^{\prime} \rightarrow Y$ is another maximally étale factorization, constructed with respect to another polarization $H^{\prime}$, the universal property of $Z$ yields the inclusion $\beta_{*}^{\prime}\left(\mathcal{O}_{Z^{\prime}}\right) \subset \beta_{*}\left(\mathcal{O}_{Z}\right)=\mathcal{F}_{1}$. Analogously, we obtain that $\mathcal{F}_{1} \subset \beta_{*}^{\prime}\left(\mathcal{O}_{Z^{\prime}}\right)$. This shows statement (4.1.1).

It follows from Fact 2.18 that $\left.f_{*}\left(\mathcal{O}_{X}\right)^{\vee}\right|_{C}$ is nef. Since $\left.\beta_{*}\left(\mathcal{O}_{Z}\right)\right|_{C}$ has degree 0 , it is a standard fact that $\left.\mathcal{Q}^{\vee}\right|_{C}$ is nef - see [CP91, prop. 1.2(8)]. On the other side, Lemma 3.1 implies that $\left.\mathcal{Q}\right|_{C}$ has no subsheaf of semi-positive degree. As a consequence, its dual $\left.\mathcal{Q}^{\vee}\right|_{C}$ has no quotient of semi-negative degree. Hartshorne's characterization [Har71] of ample vectorbundles then implies that $\left.\mathcal{Q}^{\vee}\right|_{C}$ is ample, as claimed.

Corollary 4.2. In the setup of Theorem 4.1, if $H^{\prime} \in \operatorname{Pic}(Z)$ is any polarization, and $C^{\prime} \subset Z$ an associated general complete intersection curve, then the dual of the restriction $\alpha_{*}\left(\mathcal{O}_{X}\right) /\left.\mathcal{O}_{Z}\right|_{C^{\prime}}$ is an ample vector bundle on $C^{\prime}$.

Proof. It follows from the universal property of the maximally étale factorization (4.1.1) that the maximally étale factorization of $\alpha: X \rightarrow Z$ is the identity on $Z$,

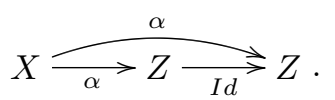

The claim then follows from Theorem 4.1(4.2.2).

Question 4.3. The Harder-Narasimhan filtration (3.0.1) of $f_{*}\left(\mathcal{O}_{X}\right)$ that is discussed on page 374 obviously depends on the choice of the line bundle $H$. As we have seen in Theorem 4.1, it turns out a posteriori that the maximally destabilizing subsheaf $\mathcal{F}_{1}$ does not depend on $H$. Are there a priori arguments to see that in our setup the maximally destabilizing subsheaf is independent of the polarization?

5. Stability under deformations, Proof of Theorem 1.7. Throughout the present section we maintain the notation and the assumptions of Theorem 1.7. Again we subdivide the lengthy proof into steps: after a reduction to the case where $f$ is finite, we prove the surjectivity of the composition morphism $\eta$ and the étalité of its lift to the normalizations separately.

5.1. Reduction to the case of a finite morphism. As an immediate consequence of the stability of Stein factorization under deformation, Proposition 2.1, we can replace $X$ with its Stein factorization. We will therefore assume without loss of generality for the remainder of the present Section 5 that $f$ is finite. 
5.2. Properness and surjectivity of the composition morphism $\eta$. The proof of surjectivity is technically a little awkward because the connected spaces $\operatorname{Hom}_{f}(X, Y)$ and $\operatorname{Hom}_{\alpha}(X, Z)$ need not be irreducible. Thus, as a first step, we show that for any irreducible component of $H \subset \operatorname{Hom}_{f}(X, Y)$ and any $\alpha^{\prime}$ with $\eta\left(\alpha^{\prime}\right) \in H$, the component $H$ is the proper image of a suitable component in $\operatorname{Hom}_{\alpha}(X, Z)$ that contains $\alpha^{\prime}$. Surjectivity and properness are then deduced in Corollaries 5.2 and 5.3 below.

Proposition 5.1. Let $f^{\prime}=\alpha^{\prime} \circ \beta \in \operatorname{Hom}_{f}(X, Y)_{\text {red }}$ be any morphism that factors via $\beta$. Further, let $H_{f^{\prime}} \subset \operatorname{Hom}_{f}(X, Y)_{\text {red }}$ be an irreducible component that contains $f^{\prime}$. Then there exists a component $H_{\alpha^{\prime}} \subset \operatorname{Hom}_{\alpha}(X, Z)_{\text {red }}$ that contains $\alpha^{\prime}$ such that $\eta\left(H_{\alpha^{\prime}}\right)=H_{f^{\prime}}$ and such that the restriction $\left.\eta\right|_{H_{\alpha^{\prime}}}$ is proper.

Proof. Let $\tilde{H}_{f^{\prime}}$ be the universal cover of a desingularization of $H_{f^{\prime}}$, and let $\tilde{f}^{\prime} \in \tilde{H}_{f^{\prime}}$ be a point that maps to $f^{\prime}$. Using that $f^{\prime}$ factors via $\beta$, we obtain the following fibered product diagram:

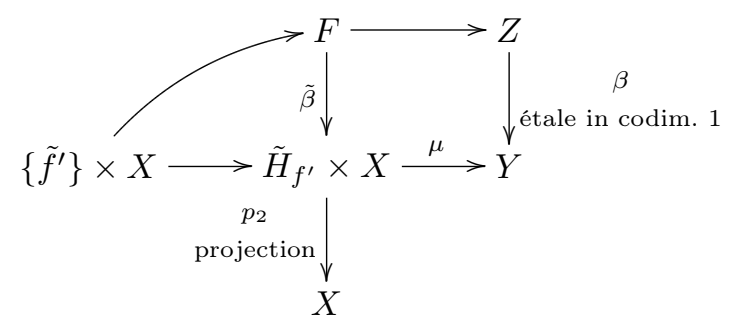

Claim 5.1.1. The morphism $\tilde{\beta}$ is also étale in codimension 1.

Proof of Claim 5.1.1. Let $R \subset Y$ be the minimal closed set $R$ such that $\beta$ is étale away from $R$. Since étale morphisms are stable under base change, we only need to show that $\tilde{R}:=\mu^{-1}(R)$ is of codimension $\geq 2$ in $\tilde{H}_{f^{\prime}} \times X$. This will be done by showing that for all $\tilde{g} \in \tilde{H}$, the intersection $\tilde{R} \cap(\{\tilde{g}\} \times X)$ is of codimension $\geq 2$ in $\{\tilde{g}\} \times X$.

To this end, let $g \in H$ be the image of $\tilde{g}$. If we identify $\{\tilde{g}\} \times X$ with $X$ in the obvious way, it is then clear that

$$
\tilde{R} \cap(\{\tilde{g}\} \times X)=g^{-1}(R) .
$$

Since $g$ is a deformation of the finite, surjective morphism $f, g$ is likewise finite and surjective, and Claim 5.1.1 follows.

As a next step in the proof of Proposition 5.1, let $F^{0}$ be the normalization of the irreducible component that contains the image of $\left\{\tilde{f}^{\prime}\right\} \times X$, and let $\tilde{\beta}^{0}: F^{0} \rightarrow \tilde{H}_{f^{\prime}} \times X$ be the obvious restriction.

Claim 5.1.2. The morphism $\tilde{\beta}^{0}$ is biholomorphic.

Proof of Claim 5.1.2. If $x \in X$ is a general point, set

$$
\tilde{H}_{x}:=p_{2}^{-1}(x) \quad \text { and } \quad F_{x}^{0}:=\left(\tilde{\beta}^{0}\right)^{-1}\left(\tilde{H}_{x}\right) \cap F^{0} .
$$

By Seidenberg's theorem [Man82], $F_{x}^{0}$ is normal, and the existence of the section $\left\{\tilde{f}^{\prime}\right\} \times X \cong X \rightarrow F^{0}$ implies that $F_{x}^{0}$ is irreducible. 
Since $\tilde{H}_{f^{\prime}} \times X$ is normal, Claim 5.1.1 now asserts that $\tilde{\beta}^{0}$ is étale in codimension 1. Since $x$ is general, this is also true for the restriction

$$
\left.\tilde{\beta}^{0}\right|_{F_{x}^{0}}: F_{x}^{0} \rightarrow \tilde{H}_{x}
$$

But because $\tilde{H}_{x}$ is smooth, Zariski-Nagata's theorem on the purity of the branch locus, [Gro71, thm. 3.1], implies that $\left.\tilde{\beta}\right|_{F_{x}}$ must in fact be étale. Since $\tilde{H}_{x}$ is simply connected, it must be isomorphic. Consequence: the finite morphism $\tilde{\beta}^{0}$ is bimeromorphic. By the analytic version of Zariski's main theorem, [Rem94, prop. 14.7], $\tilde{\beta}^{0}$ is isomorphic. This shows Claim 5.1.2.

To end the proof of Proposition 5.1, observe that Claim 5.1.2 shows the existence of a morphism $F^{0} \cong \tilde{H}_{f^{\prime}} \times X \rightarrow Z$. The universal property of the Hom-scheme thus yields a morphism $\nu: \tilde{H}_{f^{\prime}} \rightarrow \operatorname{Hom}(X, Z)_{\text {red. }}$. It follows immediately from the construction that $\nu\left(f^{\prime}\right)=\alpha^{\prime}$. Better still, we obtain a diagram

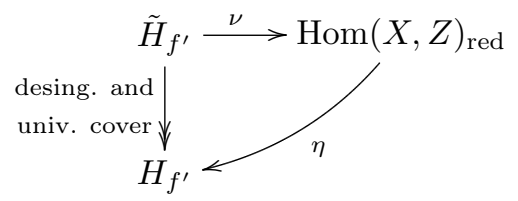

This shows that there exists a component $H_{\alpha^{\prime}}$ which contains $\alpha^{\prime}$ and surjects onto $H_{f^{\prime}}$. The properness of $\left.\eta\right|_{H_{\alpha^{\prime}}}$ follows from Diagram (5.1.3) because $\eta$ is quasi-finite. This ends the proof of Proposition 5.1.

Corollary 5.2. Let $f^{\prime}=\alpha^{\prime} \circ \beta \in \operatorname{Hom}_{f}(X, Y)_{\text {red }}$ be any morphism that factors via $\beta$. Further, let $H_{\alpha^{\prime}} \subset \operatorname{Hom}_{\alpha^{\prime}}(X, Z)_{\mathrm{red}}$ be any irreducible component that contains $\alpha^{\prime}$. Then there exists a component $H_{f^{\prime}} \subset \operatorname{Hom}_{f}(X, Y)_{\text {red }}$ that contains $f^{\prime}$ such that $\eta\left(H_{\alpha^{\prime}}\right)=H_{f^{\prime}}$ and such that the restriction $\left.\eta\right|_{H_{\alpha^{\prime}}}$ is proper.

Proof. Choose a morphism $\alpha^{\prime \prime} \in H_{\alpha^{\prime}}$ which is not contained in any other component of $\operatorname{Hom}_{\alpha^{\prime}}(X, Z)_{\text {red }}$. Now apply Proposition 5.1 to $f^{\prime \prime}=\alpha^{\prime \prime} \circ \beta$ and any component $H_{f^{\prime \prime}} \subset \operatorname{Hom}_{f}(X, Y)_{\text {red }}$ that contains $f^{\prime \prime}$. $\square$

COROllary 5.3. The morphism $\eta$ is surjective and proper.

Proof. Since $\operatorname{Hom}_{f}(X, Y)$ is connected, surjectivity of $\eta$ follows from Proposition 5.1. Since $\operatorname{Hom}_{\alpha}(X, Z)$ is connected, properness of $\eta$ follows from Corollary 5.2.

5.3. The max. étale factorization of a deformed morphism. Let $f^{\prime} \in$ $\operatorname{Hom}_{f}(X, Y)_{\text {red }}$ be any deformation of $f$. The surjectivity of $\eta$ implies that $f^{\prime}$ factor via $Z$. Here we will show that $f^{\prime}$ has $Z$ as maximally étale factorization. To this end, let

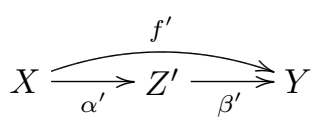

be the maximally étale factorization of $f^{\prime}$. The universal property from Definition 1.2 then yields a morphism $Z^{\prime} \rightarrow Z$. Reversing the roles of $f$ and $f^{\prime}$, we also obtain a morphism $Z \rightarrow Z^{\prime}$ which shows that $Z$ and $Z^{\prime}$ are isomorphic. 
5.4. Étalité of $\tilde{\eta}$. Since surjective and generically injective finite morphisms between normal spaces are biholomorphic, the following lemma suffices to prove that for each pair of points in the normalizations, $\tilde{\alpha}^{\prime} \in \widetilde{\operatorname{Hom}}_{\alpha}(X, Z)$ and $\tilde{f}^{\prime} \in \widetilde{\operatorname{Hom}}_{f}(X, Y)$ with $\tilde{\eta}\left(\tilde{\alpha}^{\prime}\right)=\tilde{f}^{\prime}$, the morphism $\tilde{\eta}$ induces an isomorphism of analytic neighborhoods. This shows that $\tilde{\eta}$ is étale and ends the proof of Theorem 1.7.

Lemma 5.4. Let $\alpha^{\prime}$ be a point in $\operatorname{Hom}_{\alpha}(X, Z)$ and $f^{\prime}:=\beta \circ \alpha^{\prime}$. Then there are open neighborhoods $U=U\left(\alpha^{\prime}\right)$ and $V=V\left(f^{\prime}\right)$ such that $\left.\eta\right|_{U}: U \rightarrow V$ is bijective.

Proof. Let $y \in Y$ be a general point, and $\Omega=\Omega(y)$ a sufficiently small analytic neighborhood such that

$$
\beta^{-1}(\Omega)=\Omega_{1, Z} \cup \cdots \cup \Omega_{n, Z} \quad \text { and } \quad\left(f^{\prime}\right)^{-1}(\Omega)=\Omega_{1, X} \cup \cdots \cup \Omega_{n \cdot m, X}
$$

are disjoint unions of open sets which are each isomorphic to $\Omega$. If $\Omega^{\prime} \subset \subset \Omega$ is a relatively compact neighborhood of $y$, the sets

$$
\begin{aligned}
\left(U_{i}\right)_{1 \leq i \leq n} & :=\left\{\alpha^{\prime \prime} \in \operatorname{Hom}_{\alpha}(X, Z)_{\text {red }} \mid \alpha^{\prime \prime}\left(\Omega_{1, X}^{\prime}\right) \subset \Omega_{i, Z}\right\} \\
V & :=\left\{f^{\prime \prime} \in \operatorname{Hom}_{f}(X, Y)_{\text {red }} \mid f^{\prime \prime}\left(\Omega_{1, X}^{\prime}\right) \subset \Omega\right\}
\end{aligned}
$$

are open, the $U_{i}$ are disjoint, and $\eta^{-1}(V)=\cup_{1 \leq i \leq n} U_{i}$. Using that $\left.\beta\right|_{\Omega_{i, Z}}: \Omega_{i, Z} \rightarrow \Omega$ are biholomorphic, the identity principle then immediately implies that $\left.\eta\right|_{U_{i}}: U_{i} \rightarrow V$ is injective. Proposition 5.1 implies that for any given number $i, U_{i}$ is either empty or surjects onto $V$. The proof is finished if choose $i$ such that $\alpha^{\prime} \in U_{i}$ and set $U:=U_{i}$. $\square$

6. Infinitesimal decomposition of the Hom-scheme. Theorem 1.10 asserts that a cover of $\operatorname{Hom}(X, Y)$ decomposes into a torus and deformations that are vertical with respect to the rational quotient. In this section we will show an infinitesimal version of the decomposition. We believe that this is of independent interest.

Before we formulate the result in Theorem 6.2 below, recall the following standard fact of algebraic group theory.

FACT 6.1. Let $G$ be an algebraic group. Then there exists a maximal compact Abelian subgroup, i.e., an Abelian variety $T \subset G$ which is a subgroup and such that no intermediate subgroup $T \subset S \subset G, T \neq S$, is an Abelian variety.

A maximal compact Abelian subgroup is unique up to conjugation.

The decomposition result then goes as follows.

TheOREm 6.2. Let $f: X \rightarrow Y$ be a surjective morphism between normal complexprojective varieties, and

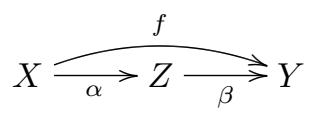

be the maximally étale factorization of $f$. Then there is a canonical decomposition of the space of infinitesimal deformations of $f$,

$$
\left.T_{\operatorname{Hom}(X, Z)}\right|_{f}=\operatorname{Hom}\left(f^{*}\left(\Omega_{Y}^{1}\right), \mathcal{O}_{X}\right)=\mathfrak{a} \oplus V,
$$

where $\mathfrak{a} \subset H^{0}\left(Z, T_{Z}\right)$ is the Lie algebra of a maximal compact Abelian variety $T \subset \operatorname{Aut}^{0}(Z)$ and where $V \subset \operatorname{Hom}\left(f^{*}\left(\Omega_{Y}^{1}\right), \mathcal{O}_{X}\right)$ is a subspace of the space of infinitesimal deformations that are vertical with respect to the maximal rationally connected fibration of $Z$. 
Recall that "infinitesimal deformations that are vertical with respect to the maximal rationally connected fibration" were defined in Definition 2.13 on page 372 .

REMARK 6.3. The functoriality of the maximal rationally chain connected fibration, [Kol96, thm. IV.5.5], implies that an infinitesimal deformation $\sigma \in$ $\operatorname{Hom}\left(f^{*}\left(\Omega_{Y}^{1}\right), \mathcal{O}_{X}\right)$ that is vertical with respect to the maximal rationally connected fibration of $Z$ is also vertical with respect to the maximal rationally connected fibration of $Y$.

Corollary 6.4. In the setup of Theorem 6.2, if $g \in \operatorname{Hom}(X, Z)_{\mathrm{red}}$, then the

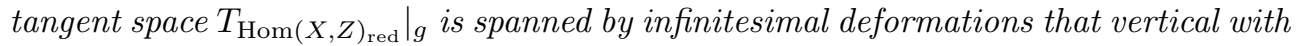
respect to the maximal rationally connected fibration of $Z$, and by tangent vectors of the T-orbit through $g$.

We prove Theorem 6.2 in the remainder of the present section. As usual, we subdivide the proof into steps.

6.1. Reduction to the case of a finite morphism. Using Stein factorization of the morphism $f$, we can assume without loss of generality that $f$-and hence $\alpha$ are actually finite. In fact, if $f$ is not finite, consider the Stein factorization as in Diagram (2.0.1) on page 368: $f=h \circ g$, where $g: X \rightarrow W_{0}$ has connected fibers and $h: W_{0} \rightarrow Y$ is finite. For the reduction, we need to show that the canonical pull-back morphism

$$
g^{*}: \operatorname{Hom}\left(h^{*}\left(\Omega_{Y}^{1}\right), \mathcal{O}_{W_{0}}\right) \rightarrow \operatorname{Hom}\left(f^{*}\left(\Omega_{Y}^{1}\right), \mathcal{O}_{X}\right)
$$

is bijective. Since $g$ is surjective, injectivity is obvious. Concerning surjectivity of this map, consider an element $u \in \operatorname{Hom}\left(f^{*}\left(\Omega_{Y}^{1}\right), \mathcal{O}_{X}\right)$,

$$
u: f^{*}\left(\Omega_{Y}^{1}\right)=g^{*} h^{*}\left(\Omega_{Y}^{1}\right) \rightarrow \mathcal{O}_{X} .
$$

The composition of the canonical map $h^{*}\left(\Omega_{Y}^{1}\right) \rightarrow g_{*} g^{*} h^{*}\left(\Omega_{Y}^{1}\right)$ and the push-forward of $u$,

$$
h^{*}\left(\Omega_{Y}^{1}\right) \rightarrow g_{*} g^{*} h^{*}\left(\Omega_{Y}^{1}\right) \stackrel{g_{*}(u)}{\longrightarrow} g_{*} \mathcal{O}_{X}=\mathcal{O}_{W_{0}}
$$

then yields a morphism $v: h^{*}\left(\Omega_{Y}^{1}\right) \rightarrow \mathcal{O}_{W_{0}}$ such that $g^{*}(v)$ and $u$ agree over the smooth part of $Y$, where the pull-back of $\Omega_{Y}^{1}$ is locally free. Since the Hom-sheaves are torsion free, this implies that $u=g^{*}(v)$.

In summary, we have shown that $a$ is an isomorphism. The reduction step is then clear.

6.2. Setup and Notation. For convenience, let $X_{\text {Sing }}, Y_{\text {Sing }}$ and $Z_{\text {Sing }}$ denote the singular loci, and set

$$
\begin{aligned}
X^{0} & :=X \backslash\left(X_{\text {Sing }} \cup \alpha^{-1}\left(Z_{\text {Sing }}\right) \cup f^{-1}\left(Y_{\text {Sing }}\right)\right) \text { and } \\
Z^{0} & :=Z \backslash\left(Z_{\text {Sing }} \cup \alpha\left(X_{\text {Sing }}\right) \cup \beta^{-1}\left(Y_{\text {Sing }}\right)\right) .
\end{aligned}
$$

Then $\operatorname{codim}_{X}\left(X \backslash X^{0}\right)=\operatorname{codim}_{Z}\left(Z \backslash Z^{0}\right) \geq 2$. The space of infinitesimal deformations can thus be rewritten as follows.

$$
\begin{aligned}
\left.T_{\operatorname{Hom}(X, Y)}\right|_{f} & =\operatorname{Hom}\left(f^{*}\left(\Omega_{Y}^{1}\right), \mathcal{O}_{X}\right) \\
& =\operatorname{Hom}\left(f^{*}\left(\Omega_{Y}^{1}\right)^{\vee}, \mathcal{O}_{X}\right)=\operatorname{Hom}\left(f^{*}\left(\Omega_{Y}^{1}\right) \mid X^{0}, \mathcal{O}_{X^{0}}\right) \\
& =H^{0}\left(X^{0}, f^{*}\left(T_{Y}\right)\right)=H^{0}\left(Z^{0}, \alpha^{*}\left(T_{Z}\right)\right) \\
& =H^{0}\left(Z^{0}, \alpha_{*}\left(\mathcal{O}_{X^{0}}\right) \otimes T_{Z^{0}}\right)=\operatorname{Hom}_{Z^{0}}\left(\alpha_{*}\left(\mathcal{O}_{X^{0}}\right)^{*}, T_{Z^{0}}\right) .
\end{aligned}
$$


Notation 6.5. If $\sigma \in H^{0}\left(X, f^{*}\left(T_{Y}\right)\right)$ is any infinitesimal deformation of the morphism $\alpha$, let $\hat{\sigma} \in \operatorname{Hom}_{Z^{0}}\left(\alpha_{*}\left(\mathcal{O}_{X^{0}}\right), T_{Z^{0}}\right)$ be the associated morphism.

Lemma 6.6. Let $\sigma$ be an infinitesimal deformation. Then

$$
\left.\sigma\right|_{X^{0}} \in H^{0}\left(X^{0}, \alpha^{*} \text { Image }(\hat{\sigma})\right) .
$$

Proof. The claim immediately follows from the definition of $\hat{\sigma}$ : if $z \in Z^{0}$ is a general point, and $\alpha^{-1}(z)=\left\{x_{i} \mid i=1 \ldots m\right\}$, then the image of $\hat{\sigma}$ at $z$ is spanned by the tangent vectors $T \alpha\left(\sigma\left(x_{i}\right)\right)_{i=1 \ldots m}$. $\square$

6.3. Decomposition of the Infinitesimal Deformations. Recall Fact 2.17 which asserts that $\alpha_{*}\left(\mathcal{O}_{X^{0}}\right) \cong \mathcal{O}_{Z^{0}} \oplus \mathcal{E}_{\alpha}^{\vee}$. This, together with the Equations (6.4.1) yields a decomposition

$$
\operatorname{Hom}\left(f^{*}\left(\Omega_{Y}^{1}\right), \mathcal{O}_{X}\right)=H^{0}\left(Z, T_{Z}\right) \oplus \underbrace{\operatorname{Hom}_{Z^{0}}\left(\mathcal{E}_{\alpha}, T_{Z^{0}}\right)}_{=: V^{\prime}} .
$$

Notation 6.7. If $\sigma \in H^{0}\left(X, f^{*}\left(T_{Y}\right)\right)$ is any infinitesimal deformation of the morphism $\alpha$, let $\hat{\sigma}^{\prime} \in H^{0}\left(Z, T_{Z}\right)$ and $\hat{\sigma}^{\prime \prime} \in \operatorname{Hom}_{Z^{0}}\left(\mathcal{E}_{\alpha}, T_{Z^{0}}\right)$ be the associated vector field and morphism, respectively.

6.4. Interpretation of $V^{\prime}$. We will now show that infinitesimal deformations $\sigma$ of $\alpha$, which correspond to elements in $V^{\prime}$ are vertical with respect to the rational quotient of $Z$. To this end, choose an ample bundle $H \in \operatorname{Pic}(Z)$ and let $C \subset Z$ be an associated general complete intersection curve. Fact 2.17 and the characterization of the maximally étale factorization, Corollary 4.2 , then assert that the restriction $\left.\mathcal{E}_{\alpha}^{\vee}\right|_{C}$ is anti-ample. It follows that $\left.\mathcal{E}_{\alpha}\right|_{C}$ is ample, and so is its image in $\left.T_{Z^{0}}\right|_{C}$ under the map $\hat{\sigma}^{\prime \prime}$. The refinement of Miyaoka's characterization of uniruled manifolds, Fact 2.15, implies that Image $\left(\hat{\sigma}^{\prime \prime}\right)$ is then vertical with respect to the rational quotient of $Z$, and Lemma 6.6 yields the claim.

6.5. The Abelian variety $T$ and end of the proof of Theorem 6.2 . We consider the connected algebraic group $\operatorname{Aut}^{0}(Z)$. By a classical theorem of Chevalley, there exists an extension

$$
0 \rightarrow L \rightarrow \operatorname{Aut}^{0}(Z) \rightarrow T^{\prime} \rightarrow 0
$$

where $L$ is linear-algebraic and $T^{\prime}$ an Abelian variety. This sequence is not necessarily split, but it is known [Lie78, thm. 3.12] that there is a maximal compact Abelian subgroup $T \subset \operatorname{Aut}^{0}(Z)$ such that the induced map $T \rightarrow T^{\prime}$ is étale.

Let $\mathfrak{a} \subset H^{0}\left(Z, T_{Z}\right)$ be the subalgebra generated by $T$ and $\mathfrak{a}^{\prime}$ that one generated by $L$. This gives a decomposition

$$
H^{0}\left(Z, T_{Z}\right)=\mathfrak{a} \oplus \mathfrak{a}^{\prime} .
$$

Since $L$ is linear-algebraic, the closures of its orbits are rationally connected. As a consequence, $L$ acts trivially on the rational quotient $Q_{Z}$, hence $\mathfrak{a}^{\prime}$ is vertical and we obtain a decomposition

$$
\operatorname{Hom}\left(f^{*}\left(\Omega_{Y}^{1}\right), \mathcal{O}_{X}\right)=\mathfrak{a} \oplus V
$$

with $V:=\mathfrak{a}^{\prime} \oplus V^{\prime}$ vertical. This ends the proof of Theorem 6.2. 
7. Decomposition of the Hom-scheme, Proof of Theorem 1.10. The proof of Theorem 1.10, which we give in this section, is the longest and most involved in this paper. Before we start with all the details in Section 7.2 below, we give a short idea of proof.

7.1. Idea of proof. In Section 7.2 we will quickly reduce to the case where $f$ is finite. For simplicity, assume further that the maximally rationally connected fibration $q_{Y}: Y \rightarrow Q_{Y}$ is a morphism and that the maximally étale factorization is an isomorphism. Let $T \subset \operatorname{Aut}^{0}(Y)$ be a maximal compact Abelian subgroup, as in Fact 6.1 above.

Under these assumptions, the composition morphism

$$
\begin{array}{ccc}
\tau: \operatorname{Hom}_{f}(X, Y) & \rightarrow & \operatorname{Hom}\left(X, Q_{Y}\right) \\
f^{\prime} & \mapsto & q_{Y} \circ f^{\prime}
\end{array}
$$

is equivariant with respect to the natural $T$-action on $\operatorname{Hom}_{f}(X, Y)$ and $\operatorname{Hom}\left(X, Q_{Y}\right)$, respectively. The infinitesimal decomposition of the Hom-scheme, Theorem 6.2, then asserts that the image of $\tau$ contains a dense $T$-orbit. By properness, the image of $\tau$ will be homogeneous under the $T$-action.

The standard fact that actions of Abelian varieties on rationally connected varieties are necessarily trivial (note that this is not true for rationally chain connected varieties!) then implies that $T$-orbits in $\operatorname{Hom}_{f}(X, Y)$ surject finitely onto the image of $\tau$, better still, that they are étale over the image of $\tau$. This quickly gives the decomposition.

The main difficulty in the proof of Theorem 1.10 is that $q_{Y}$ need not be regular. Although the space of rational maps $X \rightarrow Q_{Y}$ can easily be defined as a subscheme of $\operatorname{Hilb}\left(X \times Q_{Y}\right)$, its universal properties are too weak to construct a morphism similar to $\tau$ above - see [Han87, Han88] for a discussion of the complications that already arise with the space of birational automorphisms. We will need to consider a somewhat weaker construction instead.

7.2. Reduction to the case of a finite morphism. Using the stability of Stein factorization under deformation, Proposition 2.1, we can assume without loss of generality that the morphism $f$ is finite. Throughout, we consider the maximally étale factorization of $f$,

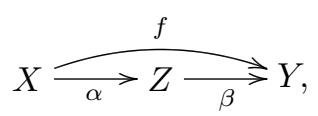

where $\beta$ is étale in codimension 1 .

7.3. Setup of notation. Before we seriously start the proof of Theorem 1.10, we need to set up some notation.

Notation 7.1. Let $q_{Z}: Z \rightarrow Q_{Z}$ and $q_{Y}: Y \rightarrow Q_{Y}$ be the maximal rationally connected fibrations and

$$
\nu: \widetilde{\operatorname{Hom}}_{f}(X, Y) \rightarrow \operatorname{Hom}_{f}(X, Y)_{\text {red }}
$$

be the normalization morphism. Let $T \subset \operatorname{Aut}^{0}(Z)$ be a maximal compact Abelian subgroup, as discussed in Fact 6.1 above.

REMARK 7.2. The group $T \subset \operatorname{Aut}^{0}(Z)$ naturally acts on $Z$ and on $\operatorname{Hom}_{\alpha}(X, Z)_{\text {red }}$. By Proposition 2.11 and the conventions fixed in Notation 2.12, 
the group $T$ acts also on the base of the maximally rationally connected fibration $Q_{Z}$. With these actions, the maximally rationally connected fibration map $q_{Z}: Z \rightarrow Q_{Z}$ is automatically $T$-equivariant wherever it is defined.

As a next step, we define subvarieties $H_{\text {vert }}^{g} \subset \operatorname{Hom}_{\alpha}(X, Z)$ which are the analogues to the fibers of the map $\tau$ that was discussed in the introductory Section 7.1 above.

Notation 7.3. If $g \in \operatorname{Hom}_{\alpha}(X, Z)$ is any morphism, define the reduced subvariety

$$
H_{\text {vert }}^{g}:=\left\{h \in \operatorname{Hom}_{\alpha}(X, Z)_{\text {red }} \mid q_{Z} \circ g=q_{Z} \circ h\right\} .
$$

As in Notation 1.9, we call $H_{\mathrm{vert}}^{g}$ the "space of relative deformations of $g$ over $q_{Z}$ ". Consider the restricted group action morphism

$$
\begin{array}{rlc}
\mu_{g}: T \times H_{\text {vert }}^{g} & \rightarrow & \operatorname{Hom}_{\alpha}(X, Z)_{\text {red }} \\
\left(t, \alpha^{\prime}\right) & \mapsto & t \circ \alpha^{\prime}
\end{array}
$$

REMARK 7.4. If $t \in T$ and $g \in \operatorname{Hom}_{\alpha}(X, Z)_{\text {red }}$ are any two elements, the associated vertical deformation spaces of $g$ and $t \cdot g$ differ only by translation in $\operatorname{Hom}_{\alpha}(X, Z)_{\mathrm{red}}$. More precisely, we have $H_{\mathrm{vert}}^{t \cdot g}=t \cdot H_{\mathrm{vert}}^{g}$. This follows trivially from the equivariance of $q_{Z}$.

7.4. Study of the restricted action morphism. The spaces $\operatorname{Hom}_{\alpha}(X, Z)_{\text {red }}$ and $H_{\mathrm{vert}}^{\alpha}$ are not necessarily proper. We will show now, however, that the restricted group action map $\mu_{\alpha}$ is still a proper morphism. This will suffice to prove both parts of Theorem 1.10 .

Proposition 7.5. The restricted action morphism $\mu_{\alpha}: T \times H_{\mathrm{vert}}^{\alpha} \rightarrow$ $\operatorname{Hom}_{\alpha}(X, Z)_{\mathrm{red}}$ is proper and surjective. It becomes étale after passing to the normalization.

Assume for the moment that Proposition 7.5 holds true. We will first show that this implies Theorem 1.10 and then, in Sections 7.6-7.7 below, prove the proposition.

Proof of Theorem 1.10, Statement (1.10.1). Let $\tilde{\mu}_{\alpha}: T \times \tilde{H}_{\mathrm{vert}}^{\alpha} \rightarrow{\widetilde{\operatorname{Hom}_{\alpha}}}_{(X, Z)}$ be the étale morphism between the normalizations that is associated with $\mu_{\alpha}$. Let

$$
\eta: \operatorname{Hom}_{\alpha}(X, Z)_{\mathrm{red}} \rightarrow \operatorname{Hom}_{f}(X, Y)_{\mathrm{red}}
$$

be the proper and surjective composition morphism discussed in Theorem 1.7, and $\tilde{\eta}$ the associated étale morphism between the normalizations. By Proposition 7.5 and Theorem 1.7, the composition

$$
\hat{\mu}_{\alpha}:=\tilde{\eta} \circ \tilde{\mu}_{\alpha}: T \times \tilde{H}_{\text {vert }}^{\alpha} \rightarrow{\widetilde{\operatorname{Hom}_{f}}}_{(X, Y)}
$$

is then surjective and étale, and it suffices to show that

$$
\left(\eta \circ \mu_{\alpha}\right)\left(\{e\} \times H_{\text {vert }}^{\alpha}\right)=\eta\left(H_{\text {vert }}^{\alpha}\right) \subset H_{\text {vert }}^{f} .
$$


For this, observe that the universal property of the maximally rationally chain connected fibration, [Kol96, IV thm. 5.5], shows the existence of a commutative diagram of dominant rational maps as follows.

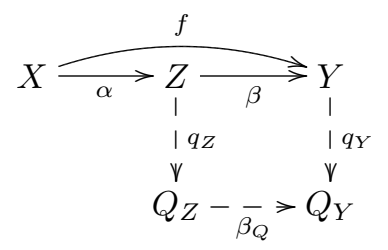

Equation (7.5.1) then follows by definition of $H$ and $H_{\text {vert }}^{\alpha}$.

Proof of Theorem 1.10, Statement (1.10.2). If $f$ is maximally étale, i.e., if $\beta$ is isomorphic, the claim follows trivially from the construction.

Now assume that $Y$ is smooth. We will see below that $H_{\text {vert }}^{\alpha}$ is a connected component of $\eta^{-1}\left(H_{\text {vert }}^{f}\right)$. It is, however, generally false that $\eta^{-1}\left(H_{\text {vert }}^{f}\right)=H_{\text {vert }}^{\alpha}$. The map $\hat{\mu}_{\alpha}=\tilde{\eta} \circ \tilde{\mu}_{\alpha}$ does therefore not always satisfy statement (1.10.2) of Theorem 1.10 and needs to be modified accordingly, by adding more components to $T \times \tilde{H}_{\text {vert }}^{\alpha}$, one for each connected component of $\eta^{-1}\left(H_{\text {vert }}^{f}\right)$. More precisely, we will show that there exists a finite set $T_{R} \subset T$, such that $\eta^{-1}\left(H_{\text {vert }}^{f}\right) \subset \operatorname{Hom}_{\alpha}(X, Z)$ is the disjoint union of copies of $H_{\text {vert }}^{\alpha}$ that are realized in $\operatorname{Hom}_{\alpha}(Z, Y)$ as translates of $H_{\text {vert }}^{\alpha}$ by elements of $T_{R}$ under the natural $T$-action on $\operatorname{Hom}_{\alpha}(Z, Y)$, i.e.

$$
\eta^{-1}\left(H_{\mathrm{vert}}^{\alpha}\right)=\bigcup_{t \in T_{R}} t \cdot H_{\mathrm{vert}}^{\alpha} .
$$

We can therefore consider the modified restricted action morphism

$$
\begin{array}{rlc}
\mu_{\alpha}^{\prime}: T \times\left(T_{R} \times H_{\mathrm{vert}}^{\alpha}\right) & \rightarrow & \operatorname{Hom}_{\alpha}(X, Z)_{\text {red }} \\
\left(t_{1},\left(t_{2}, \alpha^{\prime}\right)\right) & \mapsto & t_{1} \circ t_{2} \circ \alpha^{\prime}
\end{array}
$$

It is then obvious that the associated morphism $\tilde{\mu}_{\alpha}^{\prime}$ between normalizations is étale. Setting $\mu:=\tilde{\eta} \circ \tilde{\mu}_{\alpha}^{\prime}$ then finishes the proof.

It remains to find $T_{R}$. To this end, we need to introduce the following two subgroups of $T$.

$$
\begin{aligned}
& T_{\text {vert }, Z}:=\left\{t \in T \mid q_{Z}=q_{Z} \circ t\right\} \\
& T_{\text {vert }, Y}:=\left\{t \in T \mid \beta_{Q} \circ q_{Z}=\beta_{Q} \circ q_{Z} \circ t\right\}
\end{aligned}
$$

Claim 7.5.4. The subgroups $T_{\mathrm{vert}, Z}$ and $T_{\mathrm{vert}, Y}$ are both finite.

Proof of the claim. Since $Y$ is smooth, the quotient map $q_{Y}$ is almost holomorphic in the sense discussed in Fact 2.6. The general $q_{Y}$-fiber $Y_{q} \subset Y$ is thus smooth, rationally connected and therefore [Deb01, cor. 4.18] simply connected. Recall that $\beta$ is étale in codimension 1, i.e. étale away from a set of codimension $\geq 2$. ZariskiNagata's theorem on the purity of the branch locus, [Gro71, thm. 3.1] implies that $\beta$ is étale. The preimage $\beta^{-1}\left(Y_{q}\right)$ is then a disjoint union of several copies of the rationally connected manifold $Y_{q}$, each a fiber of $q_{Z}$. This observation has two consequences.

First, the well-known fact that actions of connected, positive-dimensional Abelian varieties on rationally connected manifolds must necessarily be trivial, [Fuj78, lem. 5.2], implies that $T_{\mathrm{vert}, Z}$ is discrete, hence finite. 
Second, the observation shows that the dominant rational map $\beta_{Q}$ defined in Diagram (7.5.2) is generically finite. This implies that $T_{\text {vert }, Y}$ is finite. Claim 7.5.4 is thus shown.

To apply Claim 7.5.4, recall from Theorem 1.7 and Proposition 7.5 that any morphism $f^{\prime} \in \operatorname{Hom}_{f}(X, Y)_{\text {red }}$ can be decomposed as $f^{\prime}=\beta \circ t \circ \alpha^{\prime}$ where $\alpha^{\prime} \in H_{\text {vert }}^{\alpha}$ and $t \in T$. We then have equivalences

$$
\begin{aligned}
& f^{\prime} \in H_{\text {vert }}^{f} \\
& \Leftrightarrow \quad q_{Y} \circ f^{\prime}=q_{Y} \circ f \quad \text { Definition } \\
& \Leftrightarrow \quad q_{Y} \circ \beta \circ t \circ \alpha^{\prime}=q_{Y} \circ \beta \circ \alpha \quad \text { Diagram 7.5.2 } \\
& \Leftrightarrow \quad \beta_{Q} \circ q_{Z} \circ t \circ \alpha^{\prime}=\beta_{Q} \circ q_{Z} \circ \alpha^{\prime} \quad \text { since } \alpha^{\prime} \in H_{\text {vert }}^{\alpha} \\
& \Leftrightarrow \quad \beta_{Q} \circ q_{Z} \circ t=\beta_{Q} \circ q_{Z} \quad \text { because } \alpha^{\prime} \text { is surjective } \\
& \Leftrightarrow \quad t \in T_{\mathrm{vert}, Y} \quad \text { Definition }
\end{aligned}
$$

This already shows that

$$
\eta^{-1}\left(H_{\mathrm{vert}}^{f}\right)=\bigcup_{t \in T_{\mathrm{vert}, Y}} t \cdot H_{\mathrm{vert}}^{\alpha}
$$

It follows immediately from the definition that two translates, $t_{1} \cdot H_{\mathrm{vert}}^{\alpha}$ and $t_{2} \cdot H_{\mathrm{vert}}^{\alpha}$ are equal if and only if $t_{1} \cdot t_{2}^{-1} \in T_{\mathrm{vert}, Z}$, and otherwise disjoint. We can therefore take $T_{R}$ to be a system of representatives for the finite group quotient $T_{\mathrm{vert}, Y} / T_{\mathrm{vert}, Z}$.

Assuming that Proposition 7.5 holds, this ends the proof of Theorem 1.10.

7.5. Proof of Proposition 7.5, a rational decomposition of the Homscheme. The aim of this section is to construct a rational analogue of the function $\tau^{\prime}$ defined in the introduction. To simplify the notation, we consider the irreducible components of $\operatorname{Hom}_{\alpha}(X, Z)_{\text {red }}$ separately.

NotaTion 7.6. Let

$$
\operatorname{Hom}_{\alpha}(X, Z)_{\mathrm{red}}=\bigcup_{i} \operatorname{Hom}_{\alpha}(X, Z)_{\mathrm{red}}^{i}
$$

be the decomposition into irreducible components, and let

$$
H_{\mathrm{vert}, i}^{g}:=H_{\mathrm{vert}}^{g} \cap \operatorname{Hom}_{\alpha}(X, Z)_{\mathrm{red}}^{i}
$$

be the associated decomposition of the $H_{\mathrm{vert}}^{g}$.

By definition of $H_{\mathrm{vert}, i}^{g}$, the space $\operatorname{Hom}_{\alpha}(X, Z)_{\mathrm{red}}^{i}$ is naturally decomposed into a disjoint union of subvarieties,

$$
\operatorname{Hom}_{\alpha}(X, Z)_{\mathrm{red}}^{i}=\bigcup_{g \in \operatorname{Hom}_{\alpha}(X, Z)_{\mathrm{red}}^{i}}^{\bullet} H_{\mathrm{vert}, i}^{g}
$$

where all subvarieties $H_{\mathrm{vert}, i}^{g}$ are all fibers of the set-theoretic map

$$
\begin{array}{ccc}
\tau_{i}^{\prime}: \operatorname{Hom}_{\alpha}(X, Z)_{\text {red }}^{i} & \rightarrow & \text { rrational maps } \left.X-\rightarrow Q_{Z}\right\} . \\
g & \mapsto & q_{Z} \circ g
\end{array}
$$

We have already discussed in Section 7.1 that it might not be possible to define a good scheme-structure on the set of rational maps which makes $\tau_{i}^{\prime}$ a morphism. 
To construct an algebraic substitute for $\tau_{i}^{\prime}$, observe that $\mathbb{C}$ is uncountable. Equation (7.6.1) therefore decomposes $\operatorname{Hom}_{\alpha}(X, Z)_{\text {red }}^{i}$ into uncountable many subvarieties. If $\overline{\operatorname{Hom}_{\alpha}(X, Z)_{\text {red }}^{i}}$ is a projective compactification of $\operatorname{Hom}_{\alpha}(X, Z)_{\text {red }}^{i}$, then Chow $\left(\overline{\operatorname{Hom}_{\alpha}(X, Z)_{\text {red }}^{i}}\right)$ has only countably many components. The decomposition of $\operatorname{Hom}_{\alpha}(X, Z)_{\text {red }}^{i}$ therefore yields a rational map between varieties

$$
\begin{array}{ccc}
\tau_{i}: \operatorname{Hom}_{\alpha}(X, Z)_{\mathrm{red}}^{i} & -\rightarrow & \operatorname{Chow}\left(\overline{\operatorname{Hom}_{\alpha}(X, Z)_{\mathrm{red}}^{i}}\right) \\
g & \mapsto & \text { closure of } H_{\mathrm{vert}, i}^{g}
\end{array}
$$

that agrees with $\tau_{i}^{\prime}$ on an open subset.

Although $\tau_{i}$ is only a rational map, there is a little that we can say about its infinitesimal structure.

Lemma 7.7. If $\operatorname{Hom}_{\alpha}(X, Z)_{\text {red }}^{i} \subset \operatorname{Hom}_{\alpha}(X, Z)_{\text {red }}$ is any irreducible component and $g \in \operatorname{Hom}_{\alpha}(X, Z)_{\mathrm{red}}^{i}$ a general point, then the kernel of the tangent morphism $\operatorname{ker}\left(\left.T \tau_{i}\right|_{g}\right)$ is exactly the space of vertical infinitesimal deformations.

In particular, the tangent space $T_{\mathrm{Hom}_{\alpha}(X, Z)_{\mathrm{red}}^{i} \mid g}$ at $g$ is spanned by the tangent space to $H_{\mathrm{vert}, i}^{g}$, and by the tangent space to the $T$-orbit through $g$.

Proof. Define a distribution, i.e. a saturated subsheaf $\mathcal{F} \subset T_{\operatorname{Hom}_{\alpha}(X, Z)_{\text {red }}^{i}}$ of $\mathcal{O}_{X^{-}}$ modules, as follows: if $h \in \operatorname{Hom}_{\alpha}(X, Z)_{\text {red }}^{i}$ is a smooth, general point, let

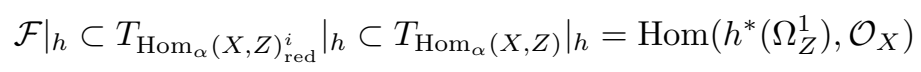

be those elements that are

- tangent to the reduced scheme $\operatorname{Hom}_{\alpha}(X, Z)_{\text {red }}^{i}$, and

- vertical with respect to the rational quotient $q_{z}: Z \rightarrow Q_{Z}$.

It is clear that $\left.\operatorname{ker}\left(\left.T \tau_{i}\right|_{g}\right) \subset \mathcal{F}\right|_{g}$. To show the other inclusion, assume that we are given a vertical infinitesimal deformation $\left.\vec{v} \in \mathcal{F}\right|_{g}$. In order to prove Lemma 7.7, we need to show that $\vec{v}$ is tangent to $H_{\mathrm{vert}, i}^{g}$. For this, consider a holomorphic arc $\gamma: \Delta \rightarrow \operatorname{Hom}_{\alpha}(X, Z)_{\text {red }}^{i}$ such that

(7.7.1) $\gamma(0)=g$, and the derivatives satisfy

(7.7.2) $\gamma^{\prime}(0)=\vec{v}$, and

(7.7.3) $\gamma^{\prime}(t) \subset \gamma^{*}(\mathcal{F})$, for all $t \in \Delta$.

The infinitesimal description of the universal morphism $\Delta \times X \rightarrow Z$ then shows that the image of $\gamma$ is entirely contained in $H_{\mathrm{vert}, i}^{g}$. It follows that $\left.\vec{v} \in T_{H_{\mathrm{ver}, i}^{g}}\right|_{g}=$ $\operatorname{ker}\left(\left.T \tau_{i}\right|_{g}\right)$.

7.6. Proof of Proposition 7.5, properness and surjectivity. With the preparations from the previous section, we can now prove the first assertion of Proposition 7.5. We show surjectivity first for the restricted action morphism $\mu_{\tilde{g}}$, where $\tilde{g}$ is a general element.

Lemma 7.8. If $\operatorname{Hom}_{\alpha}(X, Z)_{\text {red }}^{i} \subset \operatorname{Hom}_{\alpha}(X, Z)_{\text {red }}$ is any irreducible component and $\tilde{g} \in \operatorname{Hom}_{\alpha}(X, Z)_{\text {red }}^{i}$ a general point, then $\mu_{\tilde{g}}$ surjects onto $\operatorname{Hom}_{\alpha}(X, Z)_{\mathrm{red}}^{i}$.

Proof. Lemma 7.7 and the infinitesimal decomposition, Corollary 6.4, together imply that $\mu_{\tilde{g}}$ is of maximal rank at $\tilde{g}$ and therefore dominates the component $\operatorname{Hom}_{\alpha}(X, Z)_{\text {red }}^{i}$.

To show that $\mu_{\tilde{g}}$ is surjects onto $\operatorname{Hom}_{\alpha}(X, Z)_{\text {red }}^{i}$, it suffices to show that its image is closed, i.e. that that the limit of every convergent sequence of points in the image is again contained in the image. Using the compactness of $T$, this follows immediately. 
It is now easy to extend the surjectivity result to all $g \in \operatorname{Hom}_{\alpha}(X, Z)_{\text {red }}^{i}$.

LEMMA 7.9. In the setup of Lemma 7.8, if $g \in \operatorname{Hom}_{\alpha}(X, Z)_{\text {red }}^{i}$ is any point, then $\mu_{g}$ and $\mu_{\tilde{g}}$ have the same image in $\operatorname{Hom}_{\alpha}(X, Z)_{\text {red }}$.

Proof. The surjectivity of $\mu_{\tilde{g}}$, Lemma 7.8, implies that there exist elements $t \in T$ and $\hat{g} \in H_{\mathrm{vert}, i}^{\tilde{g}}$ such that $t \cdot \hat{g}=g$. By Remark 7.4, we have $H_{\mathrm{vert}}^{g}=t \cdot H_{\mathrm{vert}}^{\tilde{g}}$ and therefore

$$
\mu_{g}=t \circ \mu_{\tilde{g}} \circ\left(I d, t^{-1}\right) .
$$

This shows the claim.

Corollary 7.10. If $g \in \operatorname{Hom}_{\alpha}(X, Z)_{\text {red }}$ is any point, then $\mu_{g}$ is surjective and proper. In particular, $\mu_{\alpha}$ is surjective and proper.

Proof. The surjectivity of $\mu_{g}$ follows immediately from Lemma 7.9 and the fact that $\operatorname{Hom}_{\alpha}(X, Z)_{\text {red }}$ is connected by definition.

It remains to show that $\mu_{g}$ is proper, i.e. that the preimage of any compact set $K \subset \operatorname{Hom}_{\alpha}(X, Z)_{\text {red }}$ is again compact. But again, given a sequence $\left(t_{n}, g_{n}\right) \subset \mu_{g}^{-1}(K)$, using that $T$ is compact and the sequence $t_{n} \cdot g_{n}$ has a cumulation point in $K$, it is easy to prove that $\left(t_{n}, g_{n}\right)$ has a convergent subsequence.

7.7. Proof of Proposition 7.5, étalité. The étalité of $\tilde{\mu}_{\alpha}$ will be deduced using the following criterion. Although fairly standard, we found no reference in the literature and give a quick proof.

Proposition 7.11 (Étalité criterion). Let $f: X \rightarrow Y$ be a proper, finite morphism between irreducible varieties and assume that $Y$ is normal. If there exists a number $d \in \mathbb{N}$ such that for all $y \in Y$, the preimages $f^{-1}(y)$ contains (set-theoretically) exactly $d$ points, then $f$ is étale.

Proof. Let $y \in Y$ be any point and $f^{-1}(y)=\left\{x_{1}, \ldots, x_{d}\right\}$. By [GR84, sect. 2.3] we can find an analytic neighborhoods $U$ of $y$ and $V_{i}$ of $x_{i}$ such that $f^{-1}(U)=V_{1} \cup \cdots \cup V_{d}$ and such that the $V_{i}$ are disjoint. The restrictions $\left.f\right|_{V_{i}}: V_{i} \rightarrow U$ must then be bijective and, by the analytic version of Zariski's main theorem, [Rem94, prop. 14.7], biholomorphic. This shows the claim.

Proof of Proposition 7.5. We have already seen in Corollary 7.10 that $\mu_{\alpha}$ is proper and surjective. It remains to show that the associated morphism between the normalizations is étale.

For this, let $\widehat{\operatorname{Hom}}_{\alpha}(X, Z)_{\text {red }}$ and $\tilde{H}_{\text {vert }}^{\alpha}$ be the normalizations of $\operatorname{Hom}_{\alpha}(X, Z)_{\text {red }}$ and $H_{\text {vert }}^{\alpha}$, respectively. Further, let

$$
\tilde{\mu}_{\alpha}: T \times \tilde{H}_{\mathrm{vert}}^{\alpha} \rightarrow \widetilde{\operatorname{Hom}}_{\alpha}(X, Z)_{\mathrm{red}}
$$

be the morphism associated with $\mu_{\alpha}$. This morphism will then also be proper.

By the étalité criterion, Proposition 7.11, it remains to show that the number of elements in fibers of $\tilde{\mu}_{\alpha}$ is constant.

Recall that $T$ acts effectively and freely on $\operatorname{Hom}_{\alpha}(X, Z)_{\text {red }}$, and therefore freely on the normalization $\widetilde{\operatorname{Hom}}_{\alpha}(X, Z)_{\text {red }}$. If $G \subset T$ denotes the ineffectivity of the $T$-action on $Q_{Z}$, i.e. the kernel of the natural map $T \rightarrow \operatorname{Aut}\left(Q_{Z}\right)$, then $G$ acts freely on $H_{\text {vert }}^{\alpha}$ and $\tilde{H}_{\text {vert }}^{\alpha}$. Here we need to consider the natural $G$-action on $T \times \tilde{H}_{\text {vert }}^{\alpha}$, where $G$ acts on the factor $T$ by left multiplication. This action is likewise free. 
Proposition 7.5 is shown if we prove that for any pair $(t, \tilde{g}) \in T \times \tilde{H}_{\mathrm{vert}}^{\alpha}$, the associated $\tilde{\mu}_{\alpha}$-fiber is exactly the $G$-orbit, i.e.

$$
\tilde{\mu}_{\alpha}^{-1}\left(\tilde{\mu}_{\alpha}(t, \tilde{g})\right)=G \cdot(t, \tilde{g}) .
$$

The inclusion " $\supseteq$ " is clear.

For the other inclusion, consider two pairs contained in the same fiber,

$$
\tilde{\mu}_{\alpha}\left(t_{1}, \tilde{g}_{1}\right)=\tilde{\mu}_{\alpha}\left(t_{2}, \tilde{g}_{2}\right)
$$

If $\nu: \widetilde{\operatorname{Hom}}_{\alpha}(X, Z)_{\mathrm{red}} \rightarrow \operatorname{Hom}_{\alpha}(X, Z)_{\text {red }}$ is the normalization morphism, equation (7.11.1) then implies

$$
\nu\left(\tilde{g}_{1}\right)=t_{1}^{-1} t_{2} \cdot \nu\left(\tilde{g}_{2}\right)=\nu\left(t_{1}^{-1} t_{2} \cdot \tilde{g}_{2}\right)
$$

The assumption $\tilde{g}_{1}, \tilde{g}_{2} \in \tilde{H}_{\text {vert }}^{\alpha}$, i.e. $q_{Z} \circ \nu\left(\tilde{g}_{1}\right)=q_{Z} \circ \nu\left(\tilde{g}_{1}\right)=q_{Z} \circ \alpha$ then yields that $t_{1}^{-1} t_{2} \in G$, which ends the proof of Proposition 7.5 and hence of Theorem 1.10.

\section{REFERENCES}

[CP91] F. Campana and T. Peternell, Projective manifolds whose tangent bundles are numerically effective, Math. Ann., 289 (1991), pp. 169-187.

[Deb01] O. Debarre, Higher-dimensional algebraic geometry, Universitext, Springer-Verlag, New York, 2001.

[Fle84] H. Flenner, Restrictions of semistable bundles on projective varieties, Comment. Math. Helv., 59 (1984), pp. 635-650.

[Fuj78] A. FuJiki, On automorphism groups of compact Kähler manifolds, Invent. Math., 44 (1978), pp. 225-258.

[GHS03] T. Graber, J. Harris, and J. Starr, Families of rationally connected varieties, J. Amer. Math. Soc., 16 (2003), pp. 57-67 (electronic).

[GR84] H. Grauert and R. Remmert, Coherent analytic sheaves, Grundlehren der Mathematischen Wissenschaften [Fundamental Principles of Mathematical Sciences], vol. 265, Springer-Verlag, Berlin, 1984.

[Gro71] A. Grothendieck, Revêtements étales et groupe fondamental, Springer-Verlag, Berlin, 1971, Séminaire de Géométrie Algébrique du Bois Marie 1960-1961 (SGA 1), Dirigé par Alexandre Grothendieck. Augmenté de deux exposés de M. Raynaud, Lecture Notes in Mathematics, Vol. 224.

[Han87] M. Hanamura, On the birational automorphism groups of algebraic varieties, Compositio Math., 63 (1987), pp. 123-142.

[Han88] M. Hanamura, Structure of birational automorphism groups. I. Nonuniruled varieties, Invent. Math., 93 (1988), pp. 383-403.

[Har71] R. Hartshorne, Ample vector bundles on curves, Nagoya Math. J., 43 (1971), pp. 73-89.

[HKP06] J.-M. Hwang, S. Kebekus, and T. Peternell, Holomorphic maps onto varieties of non-negative Kodaira dimension, J. Algebraic Geom., 15 (2006), pp. 551-561.

[HM03] J.-M. Hwang AND N. MoK, Finite morphisms onto Fano manifolds of Picard number 1 which have rational curves with trivial normal bundles, J. Algebraic Geom., 12 (2003), pp. 627-651.

[HM04] J.-M. HWANG AND N. Mok, Birationality of the tangent map for minimal rational curves, Asian J. Math., 8 (2004), pp. 51-64.

[Kol92] J. Kollár (ed.), Flips and abundance for algebraic threefolds, Société Mathématique de France, Paris, 1992, Papers from the Second Summer Seminar on Algebraic Geometry held at the University of Utah, Salt Lake City, Utah, August 1991, Astérisque No. 211 (1992).

[Kol96] J. Kollár, Rational curves on algebraic varieties, Ergebnisse der Mathematik und ihrer Grenzgebiete. 3. Folge. A Series of Modern Surveys in Mathematics, vol. 32, SpringerVerlag, Berlin, 1996.

[KST07] S. Kebekus, L. SolÁ-Conde And M. Toma, Rationally connected foliations after Bogomolov and McQuillan, J. Algebraic Geom., 16 (2007), pp. 65-81. 
[Lan04] A. LANGer, Semistable sheaves in positive characteristic, Ann. of Math. (2), 159 (2004), pp. 251-276.

[Lie78] D. Liebermann, Compactness of the Chow scheme: Applications to automorphisms and deformations of Kähler manifolds, Fonctions de Plusieurs Variables Complexes III (F. Norguet, ed.), Lecture Note in Mathematics, no. 670, Springer, 1978, pp. 140-186.

[Man82] M. Manaresi, Sard and Bertini type theorems for complex spaces, Ann. Mat. Pura Appl. (4), 131 (1982), pp. 265-279.

[PS00] T. Peternell And A. J. Sommese, Ample vector bundles and branched coverings, Comm. Algebra, 28 (2000), pp. 5573-5599, With an appendix by Robert Lazarsfeld, Special issue in honor of Robin Hartshorne.

[Rem94] R. Remmert, Local theory of complex spaces, Several complex variables, VII, Encyclopaedia Math. Sci., vol. 74, Springer, Berlin, 1994, pp. 7-96. 
S. KEBEKUS AND T. PETERNELL 